\title{
Drug Use Associated Tricuspid Valve Infective Endocarditis in Pregnancy
}

\author{
Dishen Lin ${ }^{1}$, Clancy Mullan² ${ }^{2}$ Uma Deshmukh ${ }^{1}$, Mert Bahtiyar ${ }^{1}$, Hillary Hosier ${ }^{1}$, Heather \\ Lipkind $^{1}$, Sonya Abdel-Razeq ${ }^{1}$, Saurabh Rajan ${ }^{1}$, Gilead Lancaster ${ }^{1}$, and Colleen Pietras ${ }^{1}$ \\ ${ }^{1}$ Yale University School of Medicine \\ ${ }^{2}$ Yale School of Medicine
}

May 26, 2020

\begin{abstract}
We report a case of intravenous drug use-associated tricuspid valve endocarditis in a 28-year-old pregnant female at 26 -weeks gestation. Despite appropriate intravenous antibiotics, the patient developed life-threatening complications and underwent planned cesarean delivery at 28 weeks 6 days gestation followed by interval tricuspid valve replacement one week later. Both the patient and her infant were successfully managed through the perioperative period.
\end{abstract}

\section{Introduction}

Infective endocarditis (IE) requires expeditious management to prevent serious morbidity [1]. Cardiac surgery is associated with high risk of adverse outcomes in pregnancy [2]. IE during pregnancy is exceedingly rare and dangerous. Optimal management is not well described $[3,4]$. We report a patient with right-sided endocarditis at the end of her $2^{\text {nd }}$ trimester and underwent successful cesarean delivery followed by tricuspid valve replacement (TVR).

\section{Case Report}

A 28-year-old gravida 2 para 1001 female with prior uncomplicated full-term pregnancy delivered via cesarean section, active intravenous drug use (IVDU) disorder, and limited prenatal care presented at 26-weeks of gestation with fever, pleuritic chest pain, right knee pain, and dyspnea. She used cocaine and heroin the day prior and was without obstetric complaints. Fetal status was reassuring and appropriate for gestational age. Transabdominal ultrasound demonstrated an active fetus, normal amniotic fluid volume, and expected estimated fetal weight.

She was found to have Methicillin-susceptible Staphylococcus aureus (MSSA) bacteremia. Initial transthoracic echocardiogram (TTE) demonstrated a $3.5 \mathrm{~cm}$ mobile mass in the right ventricle (RV) attached to the interventricular septum and subvalvular apparatus, and trace tricuspid regurgitation (TR). Computed tomography with angiography (CTA) of the chest demonstrated multifocal cavitating pneumonia. Additionally, aspirated right knee fluid was consistent with septic arthritis.

Oxacillin, heparin infusion, and methadone were initiated with plan to undergo cesarean delivery at 34weeks of gestation followed by interval TVR after completion of a 6 -week course of oxacillin. After 5 days of treatment, repeat TTE showed enlargement of RV mass to $4.9 \mathrm{~cm}$ and severe worsening of TR (Figure 1) . Repeat arthrocentesis grew $S$. aureus, suggesting either left heart involvement or right-to-left shunting. Transesophageal echocardiogram (TEE) (Figure 2 ) identified no left-sided involvement, severe $\mathrm{TR}$, and a large patent foramen ovale (PFO). After 12 days, the patient developed a purpuric rash of her 
lower extremities, deemed as a drug-related vasculitis from oxacillin. Thus, oxacillin was replaced with IV vancomycin, resulting in gradual resolution of the rash.

Due to the urgent need for cardiac intervention, multidisciplinary consensus was to proceed with cesarean section followed by interval TVR and PFO closure. The patient received antenatal corticosteroids for fetal lung maturity. Anticoagulation was held prior to delivery. She underwent repeat cesarean delivery via classical hysterotomy at 28 weeks 6 days gestation under general anesthesia. Femoral arterial and venous access was obtained to allow for emergent availability of venoarterial extracorporeal life support (ECLS). Cardiothoracic surgery, cardiac anesthesia, obstetric anesthesia, perfusionists, and neonatology teams were present for the surgery. Fetal status remained reassuring throughout. A viable female infant was delivered weighing 1230g, with Apgar scores 3, 5, and 8 at one, five, and ten minutes, respectively. The neonate was intubated at 4 minutes of life and admitted directly to the newborn intensive care unit in stable condition.

On post-delivery day 5, patient underwent TVR via median sternotomy and right atriotomy on cardiopulmonary bypass $(\mathrm{CPB})$ and heart arrest $\mathrm{A}$ large, friable flesh-colored mass was attached to the interventricular septum and entangled throughout the anterior and posterior subvalvular apparatuses (Figure 3 ), necessitating careful excision along with removal of the anterior and posterior leaflets. The septal leaflet was free from involvement and subsequently preserved to minimize risk of injury to the atrioventricular conduction system. A 33mm Epic bioprosthetic valve was inserted. The PFO was closed primarily. Bipolar epicardial pacing wires were placed on both ventricles and right atrium. Intra-operative TEE demonstrated good biventricular function with no residual mass or interatrial shunt. Despite sparing of the septal leaflet, her post-operative course was notable for high-grade second-degree atrioventricular conduction block requiring pacemaker generator placement. She recovered well from her procedures and was discharged home in excellent condition. The neonate was extubated on day of life 10 and recovered well.

\section{Discussion}

IE is a serious condition requiring prompt treatment [2]. IV antibiotics are the preferred initial treatment, followed by surgical evaluation. Indications for early surgery include heart failure, recurrent septic emboli, and evidence of worsening infection despite appropriate antibiotic therapy.

Native valve endocarditis is most often left-sided. Isolated right-sided IE accounts for approximately $10 \%$ of cases [5]. While these infections may result from intravascular devices or underlying cardiac anomalies, $90 \%$ are related to IVDU [6]. Endocarditis during pregnancy is a rare and serious condition with poor prognosis for the mother and fetus. If surgery is needed, the timing and sequence should account for maternal and fetal clinical status, including neonatal resuscitative capacity for a given gestational age.

Valve surgery for pregnant patients should be a last resort, reserved for cases refractory to medical therapy or those with serious complications, such as our patient with a PFO and evidence of systemic embolization. While the maternal mortality risk is like that of nonpregnant women, fetal mortality may be as high as 33 percent [7], attributable to the physiologic changes on CPB. Vasoactive substances, hypotension, hypothermia, and non-pulsatile blood flow reduce uteroplacental perfusion, causing fetal hypoxia, bradycardia, and demise [8]. Fetal stress response and catecholamine surge can also increase fetal systemic vascular resistance (SVR), which can reduce fetal cardiac output and produce severe fetal respiratory acidosis [9]. Additionally, uterine contractions and subsequent preterm labor can occur during the period of rewarming [10]. Therefore, if feasible, cardiac surgery should be delayed to avoid the risks of severe prematurity for the neonate. In cases where CPB cannot be delayed until after delivery due to maternal instability, mild hypothermia with gradual rewarming is preferred.

A combined procedure of cesarean delivery followed by cardiac surgery has also been described [11]. Highdose heparinization for CPB immediately postpartum can cause severe uterine hemorrhage. Therefore, precautions must be taken including intra-abdominal wound packing, intrauterine balloon tamponade, and use of uterotonic agents.

The physiologic changes of pregnancy pose additional challenges. Maternal SVR decreases, nadiring by the 
end of the second trimester followed by a slight increase thereafter. Cardiac output increases throughout pregnancy, particularly immediately post-delivery, in part due to autotransfusion of approximately 500mL to $750 \mathrm{~mL}$ of uterine blood after placental delivery. Uterine involution after delivery decompresses the inferior vena cava, further increasing preload [11]. Although our patient was asymptomatic from severe TR, sudden hemodynamic changes following delivery may have led to rapid decompensation or sudden embolization of her massive vegetation. As such, ECLS was immediately available if she were to experience cardiovascular collapse.

In our case, the patient presented at the end of the second trimester with evidence of septic embolization to both pulmonary and systemic circulation and a large vegetation that further increased in size despite appropriate antibiotic therapy. Despite clear indications for TVR, her extremely premature gestational age was a complicating factor; surgical delay would have optimized fetal maturation. However, vegetation growth, worsening TR, and trans-PFO systemic emboli justified delivery at 28 weeks 6 days gestation followed by prompt TVR. Fortunately, both the patient and neonate survived this potentially life-threatening condition without serious complication.

\section{References}

1. Mori M, Brown K, Bin Mahmood SU, et al. Trends in infective endocarditis incidence, characteristics, and valve replacement in patients with opioid use disorders in the United States from 2005 to 2014 . $J$ Am Coll Cardiol . 2019 Mar, 73 (9 Supplement 1) 1968.

2. Wang A, Gaca JG, Chu VH. Management Considerations in Infective Endocarditis: A Review. JAMA. 2018;320(1):72-83.

3. Connolly C, O'Donoghue K, Doran H, McCarthy FP. Infective endocarditis in pregnancy: Case report and review of the literature. Obstet Med . 2015;8(2):102-104.

4. Masuda, Z., Miyamoto, Y., Une, D. et al. Acute mitral valve endocarditis at the 24th gestational week. Gen Thorac Cardiovasc Surg (2019). https://doi.org/10.1007/s11748-019-01280-7

5. Akinosoglou K, Apostolakis E, Marangos M, et al. Native valve right sided infective endocarditis. Eur J Intern Med. 2013 Sep;24(6):510-9.

6. Chahoud J, Sharif YA, Saad H, etc al. Right-Sided Infective Endocarditis and Pulmonary Infiltrates: An Update. Cardiol Rev . 2016 Sep-Oct;24(5):230-7.

7. Mahli A, Izdes S, Coskun D. Cardiac operations during pregnancy: review of factors influencing fetal outcome. Ann Thorac Surg. 2000 May;69(5):1622-6.

8. Parry AJ, Westaby S. Cardiopulmonary bypass during pregnancy.Ann Thorac Surg . 1996 Jun;61(6):1865-9.

9. Fenton KN, Heinemann MK, Hickey PR, et al. Inhibition of the fetal stress response improves cardiac output and gas exchange after fetal cardiac bypass. J Thorac Cardiovasc Surg . 1994;107:1416-22.

10. Pomini F, Mercogliano D, Cavalletti C, et al. Cardiopulmonary bypass in pregnancy. Ann Thorac Surg . 1996 Jan;61(1):259-68.

11. Tempe DK, Virmani S, Tempe A, et al. Anaesthetic management of emergency caesarean section and reoperative mitral valve replacement in a 32 weeks parturient: a case report. Ann Card Anaesth . 2002 Jan;5(1):63-7

\section{Figures}

Figure 1 Preoperative TTE showing $4.9 \mathrm{~cm}$ RV mass incorporating the subvalvular apparatus 


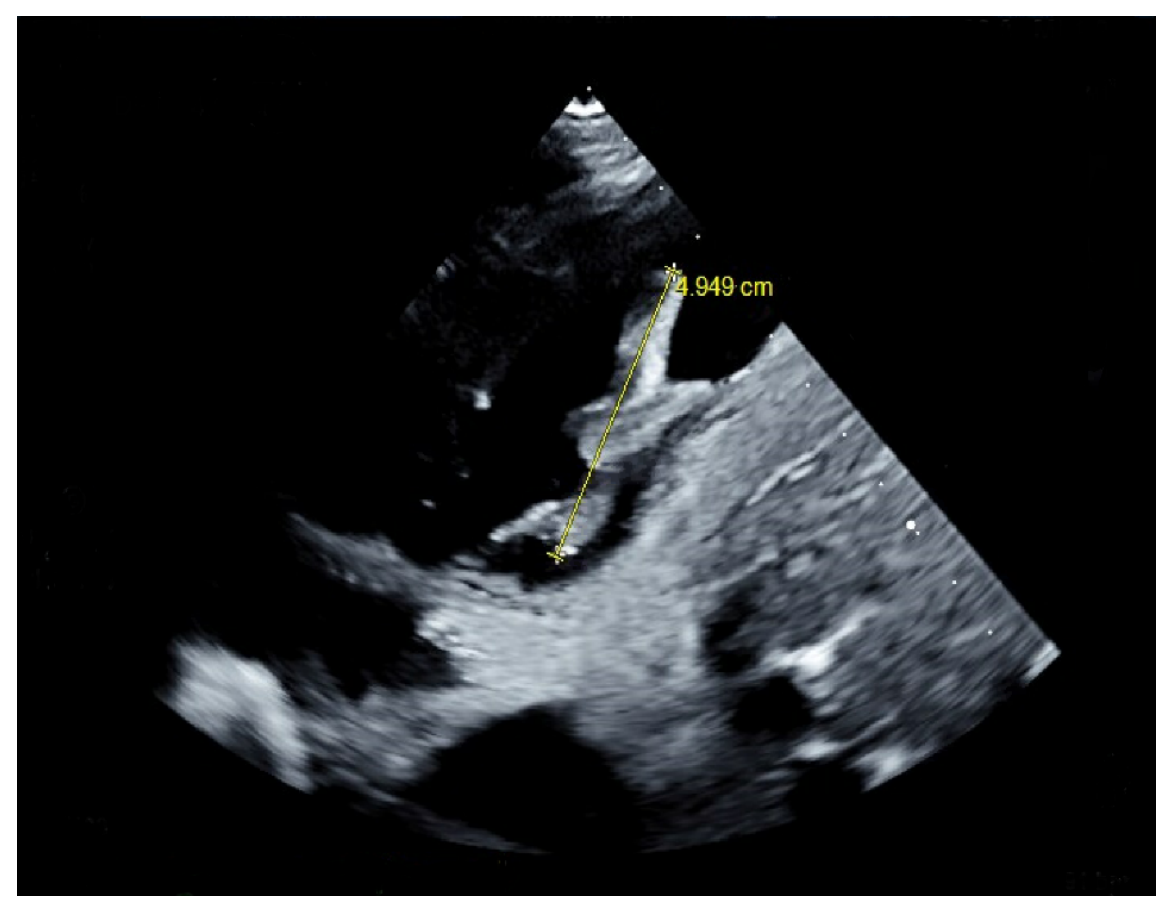

Figure 2 Preoperative TEE demonstrating (left) a in the RV and tricuspid valve and (right) torrential TR

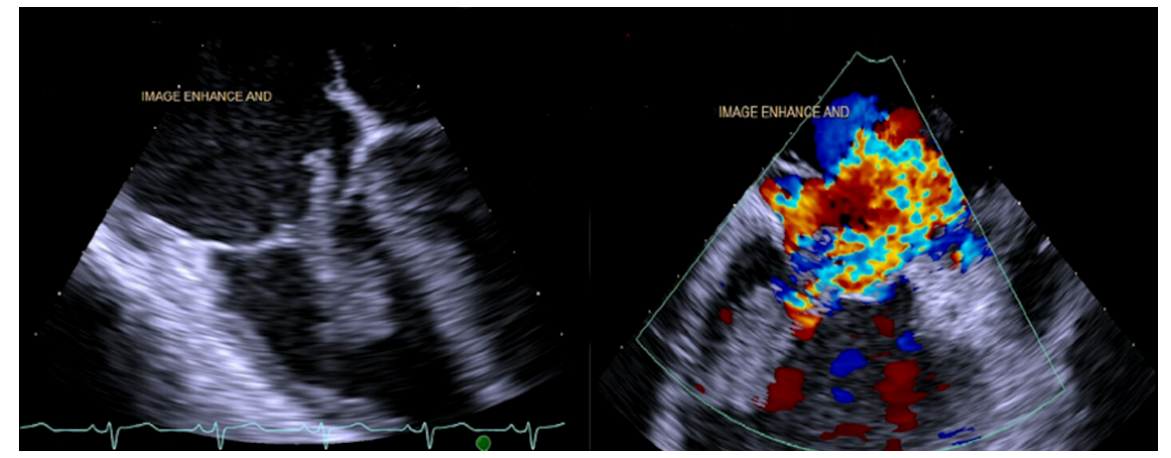

Figure 3 Massive, friable tricuspid valve vegetation post-resection 


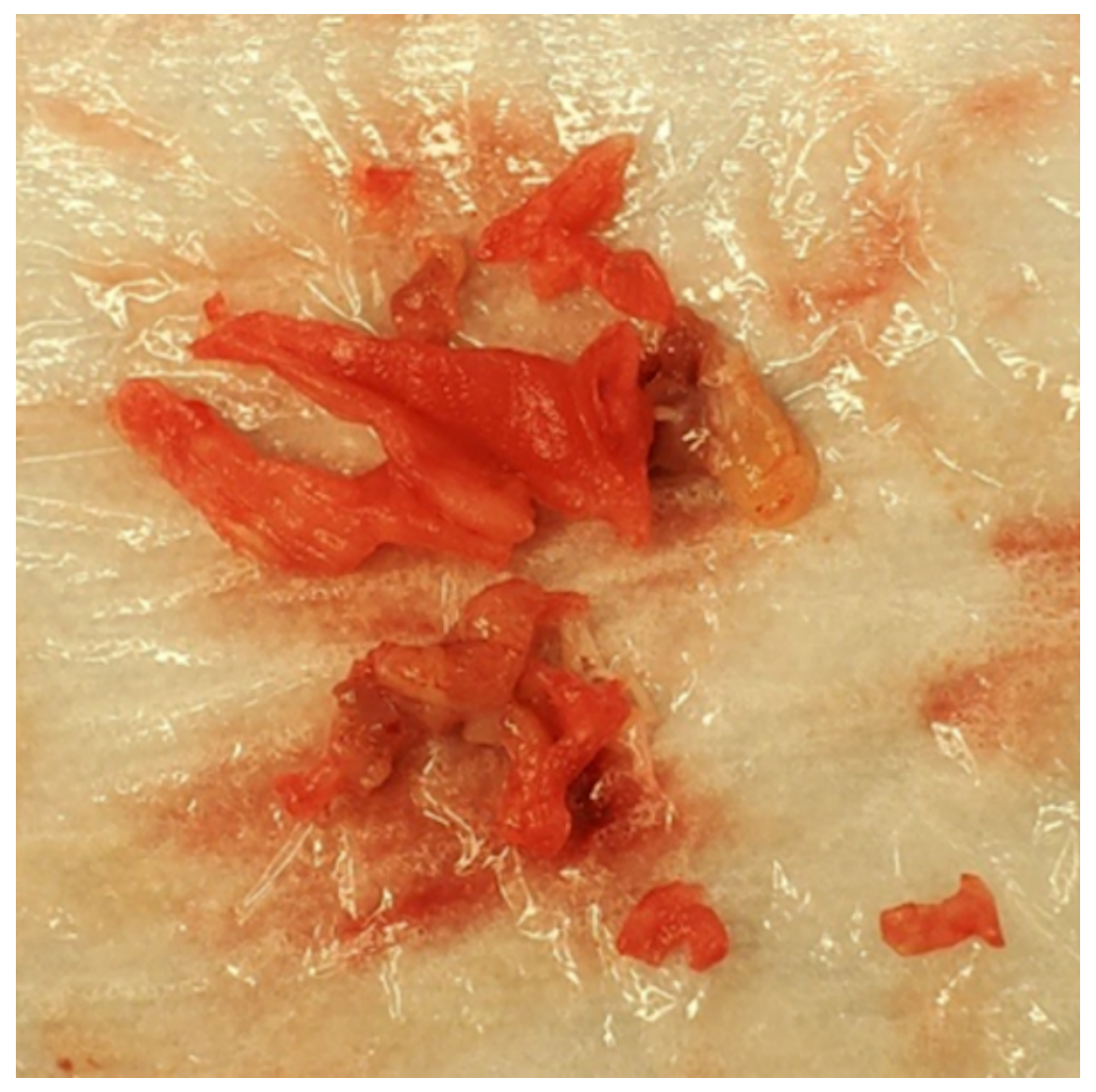

